\title{
Examination of Racial Bias in Alpha Omega Alpha Inductions: A Single-Center 15-Year Retrospective Study
}

Ruoqi Gao, Ph.D. ${ }^{1, *}$

${ }^{1}$ Northwestern University Feinberg School of Medicine, Chicago, 60611 IL, USA

This manuscript has been withdrawn by medRxiv in response to a request from Northwestern University Feinberg School of Medicine as the data collection methods used in the study constituted human subject research and the documentation was not submitted to Northwestern University's Institutional Review Board for approval. 